Provided for non-commercial research and education use. Not for reproduction, distribution or commercial use.

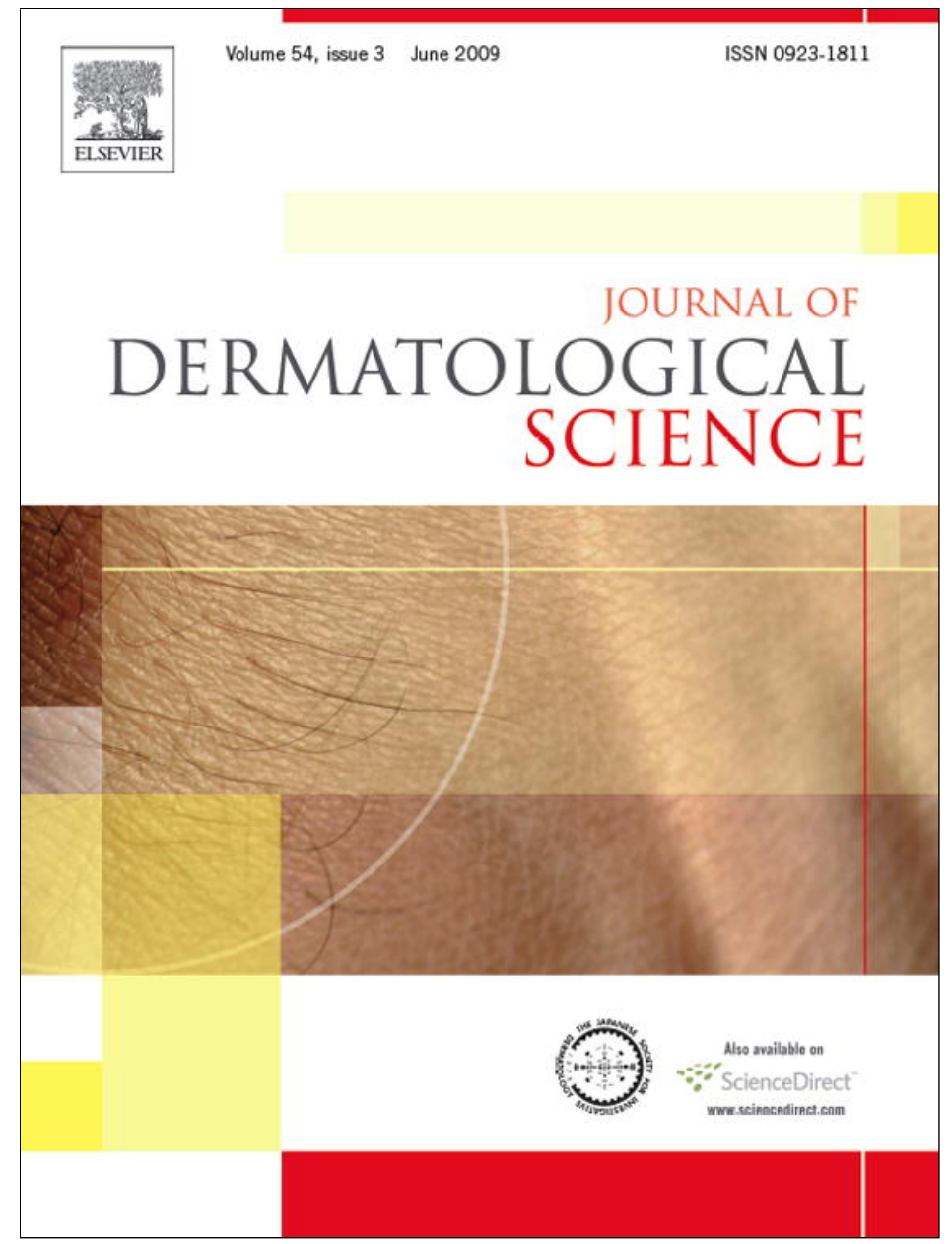

This article appeared in a journal published by Elsevier. The attached copy is furnished to the author for internal non-commercial research and education use, including for instruction at the authors institution and sharing with colleagues.

Other uses, including reproduction and distribution, or selling or licensing copies, or posting to personal, institutional or third party websites are prohibited.

In most cases authors are permitted to post their version of the article (e.g. in Word or Tex form) to their personal website or institutional repository. Authors requiring further information regarding Elsevier's archiving and manuscript policies are encouraged to visit:

http://www.elsevier.com/copyright 


\title{
Epicutaneous sensitization with a protein antigen induces Th17 cells
}

\author{
Li-Fang Wang ${ }^{a}$, Hsien-Ching Chiu ${ }^{a}$, Chih-Jung Hsu ${ }^{a}$, Ching-Yi Liu ${ }^{a}$, Yu-Han Hsueh ${ }^{\text {a }}$, Shi-Chuen Miaw ${ }^{\text {b,* }}$ \\ a Department of Dermatology, National Taiwan University Hospital and National Taiwan University College of Medicine, 100 No. 1, section 1, Ren-Ai Road, Taipei, Taiwan \\ ${ }^{\mathrm{b}}$ Graduate Institute of Immunology, National Taiwan University Hospital and National Taiwan University College of Medicine, 100 No. 1 section 1 , Ren-Ai Road, Taipei, Taiwan
}

\section{A R T I C L E I N F O}

\section{Article history:}

Received 21 July 2008

Received in revised form 16 December 2008 Accepted 19 December 2008

\section{Keywords:}

Th17 cells

Epicutaneous sensitization

Protein antigen

Allergy

\begin{abstract}
A B S T R A C T
Background: Th17 is a newly identified effector T cell lineage which plays a central role in many human inflammatory diseases and experimental animal models. Epicutaneous sensitization with a protein antigen has been proven to induce a Th2-predominant immune response and lead to development of atopic diseases in a murine protein-patch model.

Objective: We sought to assess the generation of Th17 cells in epicutaneous sensitization with a protein antigen and its regulation by environmental elements and genetic background.

Methods: BALB/c, C57BL/6, and D011.10 mice were epicutaneously immunized by patch application of the following: ovalbumin alone, or co-administration of one of TLR ligands, irritant, hapten or superantigens. IL-17 and IL-22 contents in supernatants of in vitro reactivation culture of lymph nodes cells were determined by ELISA. Frequency of IL-17-secreting CD4 T cells was measured by ELISPOT. Results: Small but significant amounts of IL-17 and IL-22 could be detected in supernatants of in vitro reactivation culture of lymph nodes cells of mice receiving patch application of ovalbumin. ELISPOT assay for IL-17 also revealed low frequency of IL-17-secreting CD4 T cells in lymph nodes cells in ovalbumin group. All TLR ligands tested including agonists for TLR2, TLR3, TLR4, TLR5, TLR7 and TLR9 as well as many environmental elements including irritant, hapten and superantigen could further promote the generation of Th17 cells. In addition, C57BL/6 mice generate less Th17 cells than BALB/c mice in epicutaneous sensitization.

Conclusion: This study demonstrates Th17 generation and its regulation by environmental elements and genetic background to a protein antigen by epicutaneous route.
\end{abstract}

(c) 2009 Japanese Society for Investigative Dermatology. Published by Elsevier Ireland Ltd. All rights reserved.

\section{Introduction}

There is no doubt that Th2 cells play a central role in the pathogenesis of atopic diseases. In asthma, Th2 cells are responsible for the development of inflammation and hyperreactivity by contributing to the differentiation and recruitment of eosinophils and mast cells, as well as production of IgE [1]. In atopic dermatitis (AD), acute skin lesions have a large number of Th2 cells, but few Th1 cells [2]. In contrast, the role Th1 cells play as effectors of atopic diseases is widely debated. Nevertheless, increasing evidence supports the notion that Th1 cells participate in the propagation of chronic inflammation and pathology of atopic diseases. For example, the frequency of Th1 cells and the level of expression of

\footnotetext{
* Corresponding author. Tel.: +886 2 223562931; fax: +886 223217921

E-mail address: smiaw@ntu.edu.tw (S.-C. Miaw).

Abbreviations: LN, lymph node; SDS, sodium dodecyl sulfate; TNBS, 2,4,6-trinitrobenzenesulfonic acid solution; SEB, staphylococcal enterotoxin B; TSLP, thymic stromal lymphopoietin; Poly(I:C), poly(inosinic-cytidylic) acid; ODN, oligodeoxynucleotide.
}

IFN- $\gamma$ from airway secretions of asthmatics appear to be positively related to the severity of disease [3]. Moreover, in a low-level challenge murine model of asthma, administration of a neutralizing Ab to IFN- $\gamma$ suppresses air hyperreactivity [4]. In AD, a shift to a Th1type cytokine milieu is always observed in chronic skin lesions [2]. Recently, a new effector T cell lineage, Th17, which produces IL-17 and IL-22, has been identified [5,6]. IL-17 is a potent proinflammatory cytokine that induces the expression of IL-1, IL-6, numerous chemokines, and recruit neutrophils [7]. Th17 is now considered to be the critical $\mathrm{T}$ effector subset responsible for mediating pathogenesis of many commonly studied models of autoimmunity and human autoimmune disorders [8]. For allergic reactions, the function of Th17 is less clear. Th17 has been shown to be a negative regulator of established allergic asthma in a murine model [9]. However, IL-17 performs important effector functions during the elicitation of contact hypersensitivity responses [10]. Studies regarding the generation of the Th17 lineage have demonstrated that IL- 6 and TGF- $\beta$ act non-redundantly and cooperatively to achieve Th17 commitment, and IL-23 functions subsequently on the committed Th17 cells to promote their expansion $[7,8]$. 
Efficient immune responses depend on a close interaction between the innate and adaptive immune systems. The innate immune system not only reacts promptly to microbial infection or environmental insult, but also instructs APCs to activate and secrete cytokines in order to polarize T cells toward an appropriate effector phenotype [11]. To date, the best characterized pattern recognition receptors which recognize pathogen-associated molecular patterns shared by many microorganisms are the TLRs. Most TLR agonists, with the exception of TLR2, stimulate the Th1 response $[11,12]$. In contrast, TLR2 ligands, as well as superantigen produced by microorganisms (e.g., staphylococcal enterotoxin B [SEB]), promote Th2 differentiation [13,14]. Thymic stromal lymphopoietin (TSLP) produced by epithelial and mast cells have also been shown to induce Th2 development [15].

Compelling evidence from clinical studies support the notion that epicutaneous sensitization is one of the important immunization routes for atopic diseases [16]. Our research group, and others have demonstrated in a murine model that epicutaneous sensitization with a protein antigen by patch application induces a predominant Th2 response with high IgE production and leads to the development of allergic asthma $[17,18]$. Epicutaneous sensitization with protein antigen has also been proven to be an important route for allergic rhinitis, and eosinophilic esophagitis in animal model $[19,20]$. Moreover, it also induces non-specific suppressor T cells to inhibit contact hypersensitivity [21]. In this study, we used a similar model to determine if Th17 cells could be induced by epicutaneous sensitization and regulated by innate elements. Moreover, the difference in Th17 induction and regulation between Th1-prone C57BL/6 mice and Th2-prone BALB/c mice was also explored.

\section{Materials and methods}

\subsection{Mice and reagents}

Eight- to twelve-week-old female BALB/c, C57BL/6, and D011.10 (OVA-specific TCR transgenic) mice were obtained from the Animal Center of the National Taiwan University College of Medicine and housed in a specific pathogen-free environment. All animal experiments were approved by the Animal Care Committee of the Medical College of National Taiwan University. Ovalbumin (OVA; grade V), 2,4,6-trinitrobenzene sulfonic acid solution (TNBS), and LPS (derived from Escherichia coli) were purchased from Sigma (St. Louis, MO, USA). Pam3CSK4, flagellin, and imiquimod (R837) were purchased from InvivoGen. Sodium dodecyl sulfate (SDS), poly(inosinic-cytidylic) acid (poly(I:C)), and thymic stromal lymphopoietin (TSLP) were purchased from Calbiochem, Amersham Bioscience, and R\&D Systems, respectively. Thioate-modified CpG-oligodeoxynucleotide (ODN-1826) was custom-synthesized by MDBio (Taipei, Taiwan). Capture and biotin-conjugated detecting Abs for IFN- $\gamma$, IL-4, and IL-5 were purchased from PharMingen. The mouse IL-13 ELISA development kit (Peprotech), IL-17A ELISA set (eBioscience), and IL-22 ELISA construction kit (Antigenix America) were used for detection of IL13, IL-17, and IL-22, respectively.

\subsection{Epicutaneous immunization}

Mice were immunized as previously described [22]. Briefly, $20 \mu \mathrm{l}$ of $100 \mathrm{mg} / \mathrm{ml}$ OVA solution was placed on the disc of a Finn chamber (Epitest). This was then applied to an area of shaved skin on the back of a mouse. For each course of immunization, freshly prepared patches were applied daily from day 1 to 5 . For groups of mice $(n=5-7)$ receiving immunization with OVA in the presence of each TLR ligand, $10 \mu \mathrm{l}$ of Pam3CSK4 $(0.5 \mu \mathrm{g} / \mu \mathrm{l})$, poly(I:C) $(2 \mu \mathrm{g} /$ $\mu \mathrm{l})$, LPS $(2 \mu \mathrm{g} / \mu \mathrm{l})$, flagellin $(0.1 \mu \mathrm{g} / \mu \mathrm{l})$, imiquimod $(2 \mu \mathrm{g} / \mu \mathrm{l})$, and CpG-ODN $(2 \mu \mathrm{g} / \mu \mathrm{l})$ were added to each Finn chamber disc. For groups of mice ( $n=5-7)$ receiving immunization with OVA in the presence of irritant, hapten, SEB, and TSLP, $10 \mu \mathrm{l}$ of 5\% SDS, $10 \mu \mathrm{l}$ of $1 \% \mathrm{TNBS}, 10 \mu \mathrm{l}$ of $0.4 \mu \mathrm{g} / \mu \mathrm{l} \mathrm{SEB}$, and $10 \mu \mathrm{l}$ of $0.01 \mu \mathrm{g} / \mu \mathrm{l} \mathrm{TSLP}$ were added, respectively.

\subsection{The measurement of cytokines in the supernatant of in vitro reactivation cultures}

Ten days after beginning the immunization course, mice were sacrificed and LN cells were pooled. $5 \times 10^{5} \mathrm{LN}$ cells were cultured in the absence or presence of $100 \mu \mathrm{g} / \mathrm{ml}$ OVA. Supernatants were harvested $48 \mathrm{~h}$ later and were stored at $-80^{\circ} \mathrm{C}$. IFN- $\gamma$, IL-5, IL-13, IL-17, and IL-22 content of supernatants were measured by a standard sandwich ELISA. The limits of detection for IL-5, IL-13, IL17 , and IL-22 were all $10 \mathrm{pg} / \mathrm{ml}$, whereas the limit of detection for IFN- $\gamma$ was $50 \mathrm{pg} / \mathrm{ml}$.

\subsection{ELISPOT assays}

A standard IL-17-specific ELISPOT assay was used. CD4 T cells were purified from LN cells by positive selection using CD4 microbeads (Miltenyi Biotec, Germany). The LN purity was $>95 \%$. $1.5 \times 10^{5}\left(1 \times 10^{4}\right.$ for D011.10) purified CD4 T cells obtained from individual mice were cultured with $3 \times 10^{5}\left(2 \times 10^{4}\right.$ for D011.10) irradiated splenocytes from naïve mice in the absence or presence of OVA $(100 \mu \mathrm{g} / \mathrm{ml})$ for $48 \mathrm{~h}$. The spots were read by an automatic immunospot analyzer (BD ParMingen). The net spot number (spot number in the absence of OVA subtracted from the spot number in the presence of OVA) was calculated.

\subsection{Quantitative real-time PCR}

OVA-TCR transgenic mice (DO11.10; three per group) were patched with PBS or OVA for 5 days. Draining LNs were obtained 10 days later. In vitro OVA reactivation cultures were grown for $48 \mathrm{~h}$. The harvested cells were purified for CD4 T cells by microbeads. Total RNA extraction, cDNA preparation, and quantitative realtime PCR were performed according to the manufacturer's instructions. The relative mRNA expression levels of each sample were normalized according to $\beta$-actin expression.

\subsection{Statistical analysis}

For each experiment, increased folds of IL-17 and IL-22 contents in mice immunized by patch application with OVA plus one of TLR ligands or environmental elements compared with mice receiving patch application with OVA only were calculated. The mean and standard deviation of increased folds were calculated from 4 to 6 independent repeated experiments. Wilcoxon rank sum test was performed for statistical analysis. $P<0.05$ was set to be significant.

\section{Results}

\subsection{Epicutaneous sensitization with a protein Ag induces a small, but significant number of Th17 cells}

We have previously shown that epicutaneous sensitization with a protein $\mathrm{Ag}$ induces a predominant $\mathrm{Th} 2 \mathrm{immune}$ response in $\mathrm{BALB} / \mathrm{c}$ mice [17]. To investigate whether Th17 cells were also induced, the IL-17 and IL-22 content in supernatants of in vitro reactivation cultures of LN cells were determined. Fig. 1A shows that small, but significant amounts of IL-17 and IL-22 could be detected in mice receiving a patch application of OVA. ELISPOT assay for IL-17 also revealed a low frequency of IL-17-secreting CD4 T cells in LN cells in the OVA group (Fig. 1B). However, we did not detect IL-17-secreting CD4 $\mathrm{T}$ cells by intracellular staining, possibly due to the low 
(A)

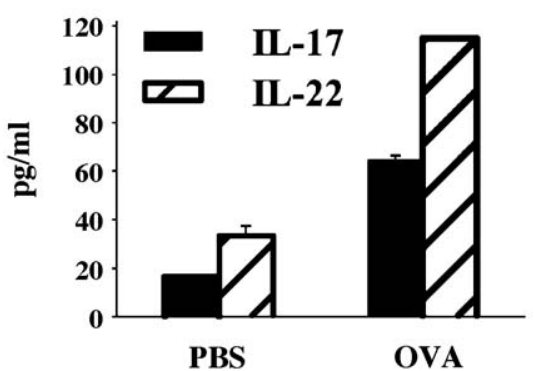

(C)

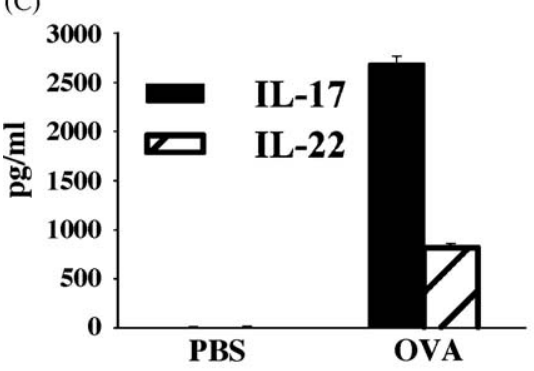

(B)

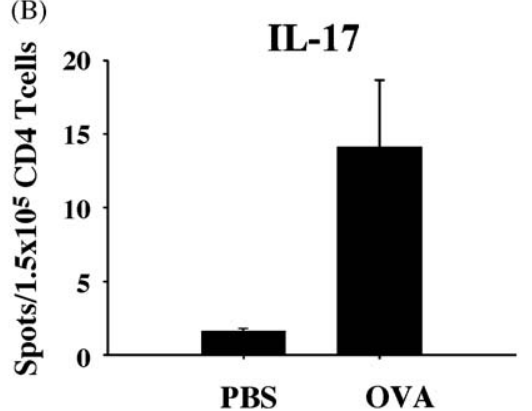

(D)

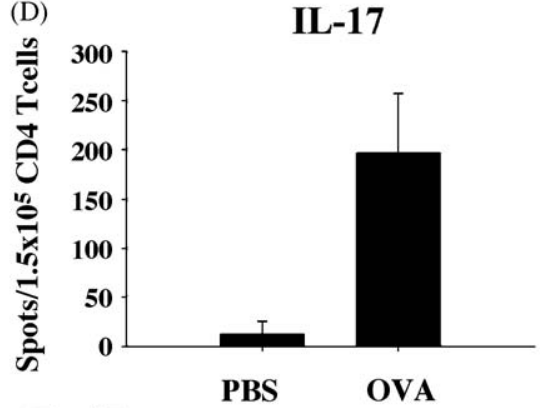

IL-17

IL-22
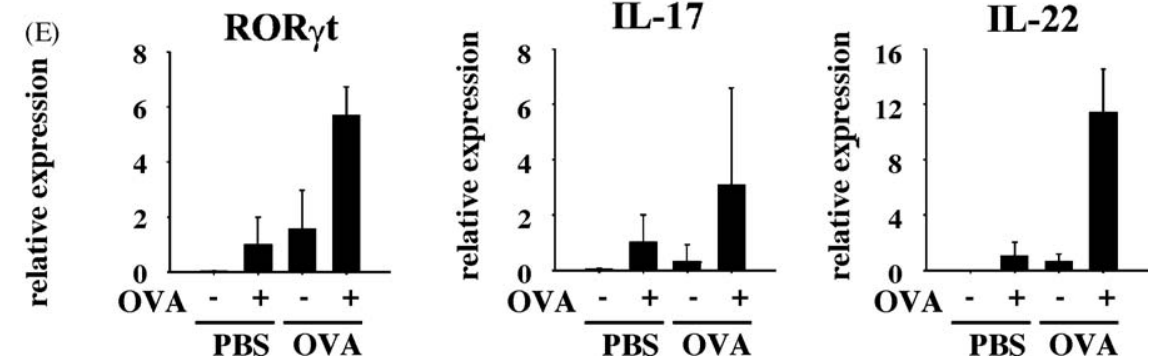

Fig. 1. Induction of IL-17 and IL-22 production and ROR $\mathrm{R}_{\gamma}$ expression by epicutaneous sensitization with OVA. Groups of BALB/C (A and B) or DO11.10 (C-E) mice were immunized by patch application with OVA solution or PBS control. Ten days later, draining LNs were obtained. (A and C) The IL-17 and IL-22 content of supernatants of in vitro reactivation cultures of the pooled draining LN cells $(n=5-7)$ were determined by ELISA. Net concentration (concentration in the absence of OVA subtracted from concentration in the presence of OVA) was presented. More than ten times independent experiments were obtained with similar results. Data from one representative experiment was shown. (B and D) CD4 T cells were purified and frequency of IL-17-secreting CD4 cells was determined by ELISPOT assay. Net frequency (frequency in the absence of OVA subtracted from frequency in the presence of OVA) was presented. (E) CD4 T cells were purified after $48 \mathrm{~h}$ in in vitro reactivation cultures, then quantitative real-time PCR was performed. The RT-PCR data shown were normalized to $\beta$-actin levels and the level of expression of OVA reactivation culture of PBS group was set at 1.0 .

frequency (data not shown). To further confirm the induction of Th17 cells by epicutaneous sensitization, OVA-specific TCR transgenic mice (DO11.10) were used. Fig. 1C shows that OVA patch application induced LN cells to secrete large amounts of IL-17 and IL22 upon in vitro restimulation with OVA. The ELISPOT assay also showed a significant frequency of IL-17-secreting CD4 T cells in LN cells (Fig. 1D). Moreover, increased mRNA levels of IL-17, IL-22, and Retinoid-related orphan receptor gamma t (ROR $\gamma \mathrm{t}$ ), a Th17-specific transcription factor, were observed in D011.10 with OVA patch applications (Fig. 1E). Since IL-6, TGF- $\beta$, and IL-23 act cooperatively to prime and maintain Th17 development, we next analyzed the mRNA expression of these cytokines in draining $L N$ of BALB/c mice by quantitative real-time PCR. We could not detect the expression of IL-6 and IL-23 in naïve mice and mice receiving OVA application (data not shown). TGF- $\beta$ expression could be detected and showed no increase after epicutaneous sensitization with OVA (data not shown). Collectively, these data demonstrate that epicutaneous sensitization with a protein $\mathrm{Ag}$ induces a small, but significant number of Th17 cells.

\subsection{TLR ligands and other environmental elements promote the induction of Th17 cells in epicutaneous sensitization}

Skin is the outermost organ exposed to many microorganisms in the environment, which are recognized as TLRs by the innate immune system of the host. Thus, we checked the effect of TLR ligands on the induction of Th17 cells in epicutaneous sensitization. We chose poly(I:C), LPS, flagellin, and CpG-ODN as representative agonists for TLR3, TLR4, TLR5, and TLR9, respectively. For TLR2 agonist, preliminary experiments using different TLR2 agonists, including purified lipoteichoic acid, zymosan, peptidoglycan, and Pam3CSK4 revealed that Pam3CSK4 was the most effective TLR2 agonist among the agonists tested (data not shown). For the TLR7 agonists, imiquimod (R873) showed a stronger effect than $E$. coli RNA/LyoVec in the preliminary studies. Thus, Pam3CSK4 and imiquimod were used in the following experiments for TLR2 and TLR7 agonists, respectively. As shown in Fig. 2A, BALB/c mice co-administered Pam3CSK4, poly(I:C), LPS, flagellin, imiquimod, or CpG-ODN with OVA generally showed a trend of increased secretion of IL-17 and IL-22 when the LN cells were in vitro-reactivated with OVA, relative to BALB/C mice receiving an OVA application only. Among all the TLR ligands tested, CpG-ODN had the strongest capability to promote IL-17 and IL-22 production. The increase reached statistical significance in Pam3CSK4, flagellin and CpG-ODN groups. There are also many kinds of environmental elements, including irritants, haptens, and superantigens secreted by microorganisms, as well as products of epithelium, which could influence Th effector production through innate immunity. We chose SDS, TNBS, SEB, and TSLP as representatives. Fig. $2 \mathrm{~B}$ shows that mice receiving epicutaneous 

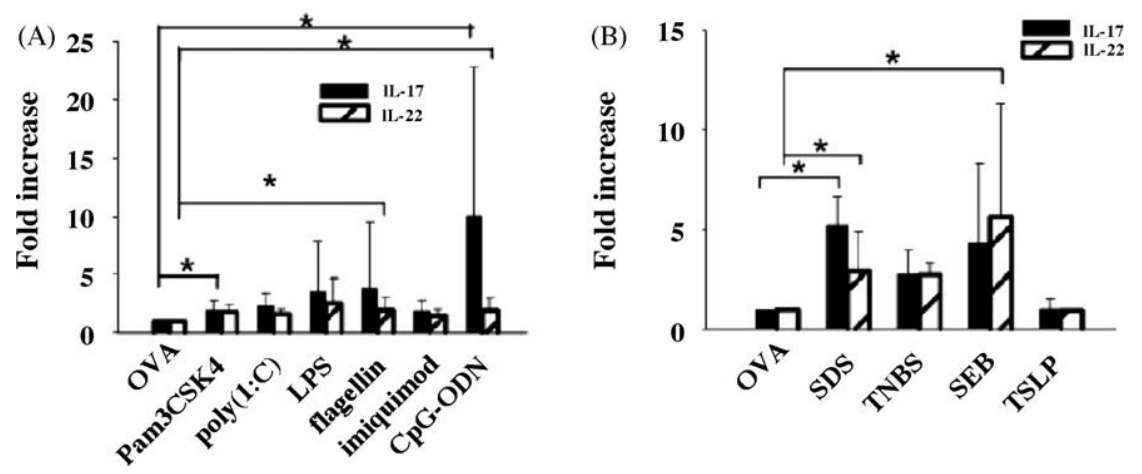

Fig. 2. Induction of Th17 cells in epicutaneous sensitization is further enhanced by TLR ligands and other environmental elements. Groups of BALB/c mice were immunized by patch application with OVA only or adding one of the followings: Pam3CSK4, poly(I:C), LPS, flagellin, imiquimod, and CPG-ODN (A), SDS, TNBS, SEB, and TSLP (B). Ten days later, cytokine contents of in vitro reactivation cultures of draining LN cells were determined by ELISA. Increased folds of IL-17 and IL-22 content compared with patch application with OVA only were shown. Pooled data were obtained from four to six independent experiments. Wilcoxon rank sum test was used for biostatistical analysis. $P<0.05\left({ }^{*}\right)$ was set to be significant.

sensitization by co-administration of SDS, TNBS, or SEB with OVA also showed a trend of higher contents of IL-17 and IL-22 in supernatants of $\mathrm{LN}$ cell reactivation culture than mice receiving OVA only. The increase reached statistical significance in SDS and SEB groups. In contrast, mice receiving epicutaneous sensitization by co-administration of TSLP with OVA showed similar levels of IL17 and IL-22 secretion with the OVA only group. The increase of IL17 and IL-22 production is not due to the direct stimulation of TLR ligands or environment elements because mice receiving patch application of either TLR ligand alone or environmental element alone showed comparable IL-17 and IL-22 levels with PBS control (data not shown). Taken together, these data suggest that many TLR ligands and environmental elements could further promote the induction of Th17 cells in epicutaneous sensitization.

\subsection{C57BL/6 mice generate fewer Th17 cells than BALB/c mice in epicutaneous sensitization}

It is generally accepted that $\mathrm{C} 57 \mathrm{BL} / 6$ and $\mathrm{BALB} / \mathrm{c}$ mice are Th1and Th2-prone mice, respectively. To explore the potential difference of Th17 generation in epicutaneous sensitization between $\mathrm{BALB} / \mathrm{c}$ and $\mathrm{C} 57 \mathrm{BL} / 6$, both strains of mice were immunized and examined simultaneously. Fig. 3A shows that OVA-patched C57BL/6 mice always generated less IL-17 and IL-22 than OVA-patched BALB/ c mice when their LN cells were in vitro-reactivated. Fig. 3B shows that the frequency of IL-17-secreting CD4 T cells in LN cells of OVApatched C57BL/6 mice was also lower than that of OVA-patched $\mathrm{BALB} / \mathrm{c}$ mice. We next checked the effects of TLR agonists and other environmental elements on Th17 generation in C57BL/6 mice. As shown in Fig. 3C, mice receiving co-administration of Pam3CSK4, poly(I:C), LPS, flagellin, imiquimod, or CpG-ODN usually produced larger amounts of IL-17 and IL-22 when compared with mice receiving OVA only. Similar to the observation in $\mathrm{BALB} / \mathrm{c}$ mice, $\mathrm{CpG}-$ ODN was also the strongest enhancer among TLR ligands for IL-17 and IL-22 production in C57BL/6 mice. The increase fold reached statistical significance in LPS and CpG-ODN groups. Co-administration of other environmental elements, represented by SDS, TNBS, SEB, or TSLP also showed a trend of increased production of IL-17 and IL-22 in LN cell reactivation cultures (Fig. 3D). The increase fold reached statistical significance in SDS group. Collectively, these results demonstrate that Th1-prone C57BL/6 mice generate fewer Th17 cells in epicutaneous sensitization with protein antigens than Th2-prone BALB/c mice.

\section{Discussion}

In this study, we demonstrated the induction of Th17 cells by epicutaneous sensitization with protein antigens in mice. This
Th17 induction is further enhanced by many environmental elements, including products of microorganisms, irritants, and haptens. Furthermore, the difference of Th17 induction in epicutaneous sensitization between Th1-prone C57BL/6 and Th2-prone BALB/c mice was also demonstrated.

Th17 is a newly identified effector of $\mathrm{T}$ cell lineage which is involved in the pathophysiology of many inflammatory autoimmune or allergic diseases [7]. The differentiation of Th17 cells was intensively investigated recently and studies revealed the crucial roles of TGF- $\beta$ and IL- 6 in its development, and the antagonizing effects of products of Th1 and Th2 lineages. Most of these data were obtained from the in vitro systems. There are far fewer reports discussing the development of Th17 cells in in vivo systems. Albanesi et al. [23] and He et al. [10] reported that epicutaneous hapten sensitization induces the development of CD4 and CD8 T cells that produce IL-17, respectively. Th17 cells could be demonstrated in a conventional EAE model by subcutaneous immunization of peptides in emulsion of CFA with intravenous injection of pertusiss toxin, which has been shown to promote Th17 generation [24,25]. For microorganisms, infection with Mycobacterium tuberculosis by the aerosol route and with Candida albicans by the intravenous route has been reported to induce Th17 development [26,27]. Very recently, He et al. reported Th17 generation by epicutaneous sensitization in $\mathrm{BALB} / \mathrm{c}$ mouse [28]. The induced Th17 response could drive airway inflammation after inhalation challenge. Their experimental protocol was different from ours in two ways. Firstly, they sensitized mice for 3 courses. Their mice had a total of three 1-week exposure to patch separated from each other by 2 -week interval. In contrast, our mice received patch application for 5 successive days only. Secondly, they performed tape stripping to remove keratin before patch application but we did not. Thus, this report which shows Th17 generation by epicutaneous sensitization with protein antigens, without the help of any adjuvant or manipulations is a pioneer demonstration of Th17 induction under physiological conditions. Considering the enhancing effects of environment elements on Th17 generation demonstrated in our current study, it is reasonable that the Th17 response induced in He's study was much stronger than ours.

In the current study, IL-17-secreting T cells were detected by intracellular staining only by in vitro PMA activation in D011.10 transgenic mice (data not shown). IL-17-secreting T cells could not be detected by intracellular staining by in vitro OVA activation in D011.10 transgenic mice or in vitro by PMA or OVA activation in BALB/c mice (data not shown). The most likely reason is the low frequency of IL-17-secreting $\mathrm{T}$ cells which was estimated to be around $0.01 \%$ by ELISPOT results in BALB/c mice. TSLP has a weak enhancing effect on Th17 induction in C57BL/6 mice, but not in $\mathrm{BALB} / \mathrm{c}$ mice. The reason is obscure at present. TSLP is generally 

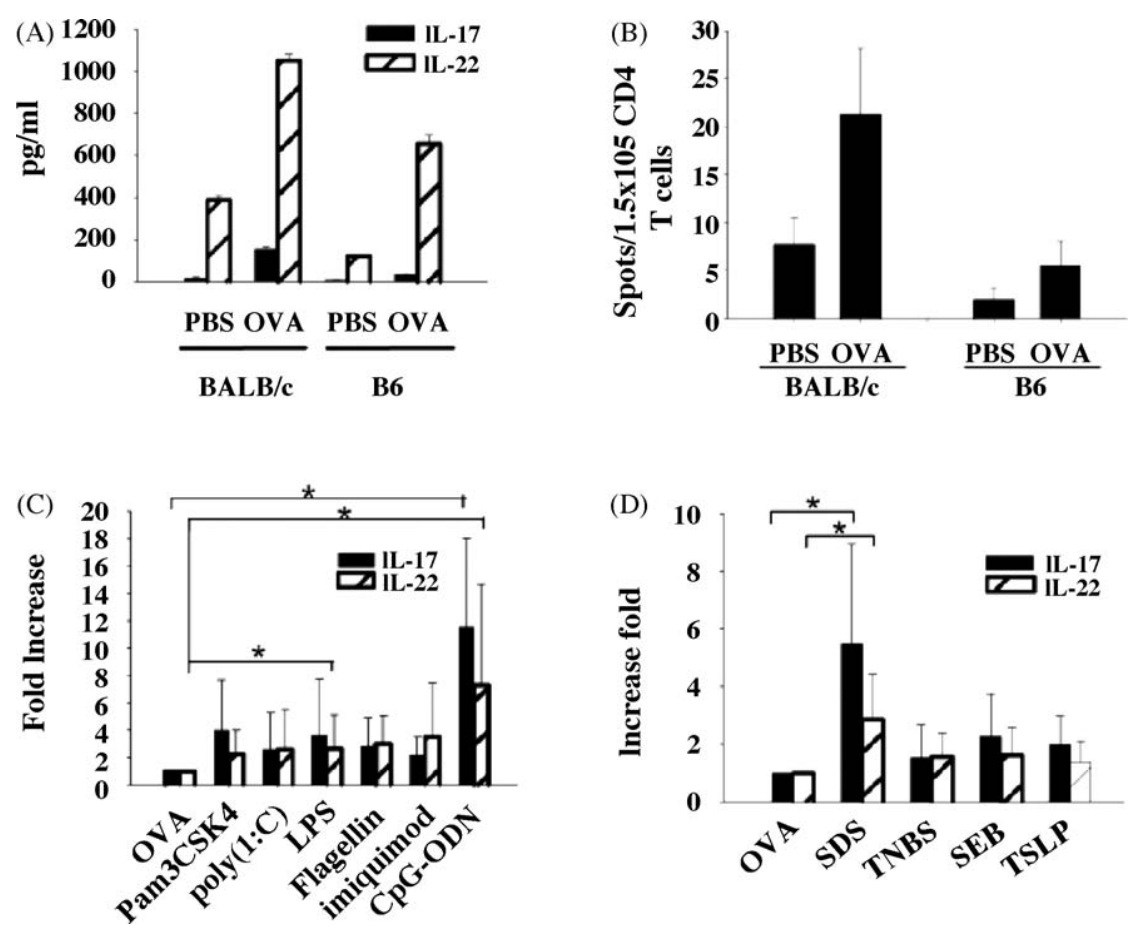

Fig. 3. The generation and regulation of Th17 cells by epicutaneous sensitization in C57BL/6 mice. (A and B) Groups of BALB/C and C57BL/6 mice were simultaneously immunized by patch application with OVA and PBS control. Ten days later, draining LNs were obtained. The IL-17 and IL-22 content of supernatants of in vitro reactivation cultures were determined by ELISA. Net concentration was presented. Three independent experiments were performed with similar results. Data from one representative experiment was shown (A). CD4 T cells were purified from draining LN cells and the frequency of IL-17-secreting CD4 T cells was determined by ELISPOT assay (B). (C and D) C57BL/6 mice received similar immunization and analysis as in Fig. 2. Pooled data from four to six independent experiments were shown. Wilcoxon rank sum test was used for biostatistical analysis. $P<0.05\left({ }^{*}\right)$ was set to be significant.

believed to be a Th2-enhancer. Thus, the Th1- and Th2-prone innate character of $\mathrm{C} 57 \mathrm{BL} / 6$ and $\mathrm{BALB} / \mathrm{c}$ mice might be a possible mechanism to explain the differential effect of TSLP in these two mouse strains.

The physiologic and pathologic significance of induction of Th17 cells by epicutaneous sensitization with protein antigens are still unclear. IL-17-producing CD4 and CD8 T cells have been shown to be important effectors for sensitization and the elicitation phase of contact hypersensitivity which was a prototype animal model of human contact dermatitis [29]. IL-17 and IL-22 cooperatively enhances expression of antimicrobial peptides by keratinocytes, thus regulating cutaneous host defense [30]. Moreover, IL-22 was recently reported to mediate IL-23-induced dermal inflammation and epidermal acanthosis in psoriasis, a common chronic cutaneous inflammatory disorder [31]. For AD, IL17 expression is enhanced in acute, but not chronic lesions [32]. Very recently, Koga et al. reported that the number of Th17 cells is increased in the peripheral blood as well as acute lesional skin and correlated with seventy of AD [33]. It was suggested that Th17 cells participate in the development of $\mathrm{AD}$ as an enhancer, but not an immune-polarizer, of AD [33]. Taken together, Th17 cells with their cytokine products may play important roles in the pathogenesis of many cutaneous inflammatory diseases.

Information regarding the reciprocal interaction between Th1/Th17 and Th2/Th17 was obtained from both in vitro and in vivo systems. In in vitro culture systems, antibodies to IFN- $\gamma$ and IL-4 should be added in order to skew T cells to Th17 differentiation, thus leading to the conclusion that products of Th1 and Th2 lineage antagonize the development of Th17. For in vivo animal model systems, most investigators reported similar observations. For example, Ab to IFN- $\gamma$ worsened EAE and adjuvant arthritis which Th17 cells play a central role in pathogenesis, whereas IFN- $\gamma$ administration protected the mice from disease development [8]. T-bet-deficient mice were also reported to show increased Th17 differentiation and increased neutrophilic airway inflammation [34]. Notably, to our knowledge, there is still no report discussing Th17 development in Th2lineage-deficient mice. For the effect of Th17 on Th1 and Th2 development, Nakae et al. [29] reported that contact and delayed-type hypersensitivities were both significantly reduced in IL-17-deficient mice due to impairment of sensitization of antigen-specific Th1 and Th2 cells. As to airway hypersensitivity response, they showed that OVA/alumn-induced airway hypersensitivity response developed normally in IL-17-deficient mice, although IL- 4 and IL- 5 production by T cells and Ab production to OVA were reduced. They attributed it to an excess Th2 response induced by alum adjuvant because airway hypersensitivity response induced by OVA/PBS inhalation was suppressed in IL$17^{-I-}$ D011.10 Tg mice [29]. For immune response modifiers, PGE2, a conventional Th2 enhancer, was shown to promote Th17 development in experimental inflammatory bowel disease and in Bordetella infection of macrophages [35,36]. Dectin-1 agonist was recently demonstrated to be an adjuvant for both Th1 and Th17 priming [24]. Epicutaneous sensitization with protein antigens induces a predominant Th2 immune response in both $\mathrm{BALB} / \mathrm{c}$ and C57BL/6 mice [17]. However the Th2 immune response induced in $\mathrm{C} 57 \mathrm{BL} / 6$ mice is always weaker when compared with BALB/C mice. The induction of Th17 cells in BALB/c and B6 mice follows similar trend. Moreover, all TLR ligands tested and many environmental elements, with either Th1 or Th2 enhancing capability, promote Th17 cells as well as Th2 immune response in our model system (data not shown). Thus, it is likely that the amplitude of Th17 cells induced by epicutaneous sensitization is determined by the intensity of the entire immune response, but not by the balance between Th1/Th2/Th17 development. In conclusion, the interaction of Th1/Th17 and Th2/Th17 is much more complicated than simple antagonism and needs further observation and investigation. 
In summary, the demonstration of Th17 induction and its regulation in epicutaneous sensitization with a protein antigen highlights its importance as an allergen sensitization route for atopic diseases, and provides crucial information for prevention of allergen sensitization and vaccine development.

\section{Acknowledgements}

This work was supported by grant from National Health Research Institutes (NHRI-EX95-9516SC) from National Taiwan University Hospital (NTUH 97S-815) and National Science Council (NSC95-2320-B-002).

\section{References}

[1] Herrick CA, Bottomly K. To respond or not to respond: T cells in allergic asthma. Nat Rev Immunol 2003;3:405-12.

[2] Leung DY, Boguniewicz M, Howell MD, Nomura I, Hamid QA. New insights into atopic dermatitis. J Clin Invest 2004;113:651-7.

[3] Busse WW, Lemanske Jr RF. Asthma. N Engl J Med 2001;344:350-62.

[4] Kumar RK, Webb DC, Herbert C, Foster PS. Interferon-gamma as a possible target in chronic asthma. Inflamm Allergy Drug Targets 2006;5:253-6.

[5] Harrington LE, Hatton RD, Mangan PR, Turner H, Murphy TL, Murphy KM, et al. Interleukin 17-producing CD4 + effector T cells develop via a lineage distinct from the T helper type 1 and 2 lineages. Nat Immunol 2005;6:1123-32.

[6] Park H, Li Z, Yang XO, Chang SH, Nurieva R, Wang YH, et al. A distinct lineage of CD4 $\mathrm{T}$ cells regulates tissue inflammation by producing interleukin 17 . Nat Immunol 2005;6:1133-41.

[7] Weaver CT, Hatton RD, Mangan PR, Harrington LE. IL-17 family cytokines and the expanding diversity of effector $\mathrm{T}$ cell lineages. Annu Rev Immunol 2007;25:821-52.

[8] Steinman L. A brief history of $\mathrm{T}(\mathrm{H}) 17$, the first major revision in the $\mathrm{T}(\mathrm{H}) 1$ / $\mathrm{T}(\mathrm{H}) 2$ hypothesis of T cell-mediated tissue damage. Nat Med 2007;13:139-45.

[9] Schnyder-Candrian S, Togbe D, Couillin I, Mercier I, Brombacher F, Quesniaux $\mathrm{V}$, et al. Interleukin-17 is a negative regulator of established allergic asthma. J Exp Med 2006;203:2715-25.

[10] He D, Wu L, Kim HK, Li H, Elmets CA, Xu H. CD8+ IL-17-producing T cells are important in effector functions for the elicitation of contact hypersensitivity responses. J Immunol 2006:177:6852-8.

[11] Mazzoni A, Segal DM. Controlling the Toll road to dendritic cell polarization. J Leukoc Biol 2004;75:721-30.

[12] Agrawal S, Agrawal A, Doughty B, Gerwitz A, Blenis J, Van Dyke T, et al. Cutting edge: different Toll-like receptor agonists instruct dendritic cells to induce distinct Th responses via differential modulation of extracellular signal-regulated kinase-mitogen-activated protein kinase and c-Fos. J Immunol 2003;171:4984-9.

[13] Redecke V, Hacker H, Datta SK, Fermin A, Pitha PM, Broide DH, et al. Cutting edge: activation of Toll-like receptor 2 induces a Th2 immune response and promotes experimental asthma. J Immunol 2004;172:2739-43.

[14] Liu T, He SH, Zheng PY, Zhang TY, Wang BQ Yang PC. Staphylococcal enterotoxin B increases TIM4 expression in human dendritic cells that drives naive CD4 T cells to differentiate into Th2 cells. Mol Immunol 2007;44:3580-7.

[15] Soumelis V, Reche PA, Kanzler H, Yuan W, Edward G, Homey B, et al. Human epithelial cells trigger dendritic cell mediated allergic inflammation by producing TSLP. Nat Immunol 2002;3:673-80.

[16] Berard F, Marty JP, Nicolas JF. Allergen penetration through the skin. Eur J Dermatol 2003;13:324-30.

[17] Wang LF, Lin JY, Hsieh KH, Lin RH. Epicutaneous exposure of protein antigen induces a predominant Th2-like response with high IgE production in mice. J Immunol 1996;156:4077-82.
[18] Spergel JM, Mizoguchi E, Brewer JP, Martin TR, Bhan AK, Geha RS. Epicutaneous sensitization with protein antigen induces localized allergic dermatitis and hyperresponsiveness to methacholine after single exposure to aerosolized antigen in mice. J Clin Invest 1998;101:1614-22.

[19] Akei HS, Brandt EB, Mishra A, Strait RT, Finkelman FD, Warrier MR, et al. Epicutaneous aeroallergen exposure induces systemic Th2 immunity that predisposes to allergic nasal response. J Allergy Clin Immunol 2006;118: 62-9.

[20] Akei HS, Mishra A, Blanchard C, Rothenberg ME. Epicutaneous antigen exposure primes for experimental eosinophilic esophagitis in mice. Gastroenterology 2005;129:985-94.

[21] Szczepanik M, Bryniarski K, Tutaj M, Skrzeczynska J, Askenase PW, Ptak W. Epicutaneous immunization induces alphabeta T-cell receptor CD4 CD8 double-positive non-specific suppressor T cells that inhibit contact sensitivity via transforming growth factor-beta. Immunology 2005;115(1):42-54.

[22] Wang LF, Hsu CJ, Miaw SC, Chiu HC, Liu CY, Yu HS. Cross-priming with an epicutaneously introduced soluble protein antigen generates Tc1 cells. Eur J Immunol 2006;36:2904-11.

[23] Albanesi C, Cavani A, Girolomoni G. IL-17 is produced by nickel-specific T lymphocytes and regulates ICAM-1 expression and chemokine production in human keratinocytes: synergistic or antagonist effects with IFN-gamma and TNF-alpha. J Immunol 1999;162:494-502.

[24] Li MO, Wan YY, Flavell RA. T cell-produced transforming growth factor-beta1 controls $\mathrm{T}$ cell tolerance and regulates Th1- and Th17-cell differentiation. Immunity 2007;26:579-91.

[25] Chen X, Howard OM, Oppenheim JJ. Pertussis toxin by inducing IL-6 promotes the generation of IL-17-producing CD4 cells. J Immunol 2007;178:6123-9.

[26] Khader SA, Bell GK, Pearl JE, Fountain JJ, Rangel-Moreno J, Cilley GE, et al. IL-23 and IL-17 in the establishment of protective pulmonary CD4 + T cell responses after vaccination and during Mycobacterium tuberculosis challenge. Nat Immunol 2007;8:369-77.

[27] Leibundgut-Landmann S, Gross O, Robinson MJ, Osorio F, Slack EC, Tsoni SV, et al. Reis ESC. Syk- and CARD9-dependent coupling of innate immunity to the induction of $\mathrm{T}$ helper cells that produce interleukin 17. Nat Immunol 2007;8:630-8.

[28] He R, Oyoshi MK, Jin H, Geha RS. Epicutaneous antigen exposure induces a Th17 response that drives airway inflammation after inhalation challenge. Proc Natl Acad Sci USA 2007;104:15817-22.

[29] Nakae S, Komiyama Y, Nambu A, Sudo K, Iwase M, Homma I, et al. Antigenspecific $T$ cell sensitization is impaired in IL-17-deficient mice, causing suppression of allergic cellular and humoral responses. Immunity 2002; 17:375-87.

[30] Liang SC, Tan XY, Luxenberg DP, Karim R, Dunussi-Joannopoulos K, Collins M, et al. Interleukin (IL)-22 and IL-17 are coexpressed by Th17 cells and cooperatively enhance expression of antimicrobial peptides. J Exp Med 2006;203:2271-9.

[31] Zheng Y, Danilenko DM, Valdez P, Kasman I, Eastham-Anderson J, Wu J, et al. Interleukin-22, a T(H)17 cytokine, mediates IL-23-induced dermal inflammation and acanthosis. Nature 2007:445:648-51.

[32] Toda M, Leung DY, Molet S, Boguniewicz M, Taha R, Christodoulopoulos P, et al. Polarized in vivo expression of IL-11 and IL-17 between acute and chronic skin lesions. J Allergy Clin Immunol 2003;111:875-81.

[33] Koga C, Kabashima K, Shiraishi N, Kobayashi M, Tokura Y. Possible pathogenic role of Th17 cells for atopic dermatitis. J Invest Dermatol 2008;128:2625-30.

[34] Fujiwara M, Hirose K, Kagami S, Takatori H, Wakashin H, Tamachi T, et al. T-bet inhibits both TH2 cell-mediated eosinophil recruitment and TH17 cellmediated neutrophil recruitment into the airways. J Allergy Clin Immunol 2007;119:662-70.

[35] Sheibanie AF, Yen JH, Khayrullina T, Emig F, Zhang M, Tuma R, et al. The proinflammatory effect of prostaglandin E2 in experimental inflammatory bowel disease is mediated through the IL-23 $\rightarrow$ IL-17 axis. J Immunol 2007; 178:8138-47.

[36] Siciliano NA, Skinner JA, Yuk MH. Bordetella bronchiseptica modulates macrophage phenotype leading to the inhibition of CD4 + T cell proliferation and the initiation of a Th17 immune response. J Immunol 2006;177:7131-8. 